\title{
Neurotrophins in Preterm Neonates: Clinical Significance in Prematurity-Related Complications and Role in Novel Cellular Therapies
}

Alicja Zawiślak*, Zofia Litwińska*, Kamila Szumilas, Dorota Gródecka-Szwajkiewicz, Bogusław Machaliński†

\begin{abstract}
The development and function of nervous system is orchestrated by various growth factors, including neurotrophic factors. Among these are classical neurotrophins, which play important roles during foetal growth and development. Neurotrophins concentrations differ between preterm and full-term neonates; in fact, these differences may be clinically significant in the development of prematurity-related complications. Novel cellular therapies are aimed at providing stem/progenitor cells, able to produce beneficial neurotrophins, to the preterm infant in order to ameliorate detrimental effects of prematurity. In this review we focus on neurotrophins significance in preterm birth complications and their possible role in paracrine effects of stem/progenitor cells applied to preterm infants.
\end{abstract}

\section{Keywords}

Neurotrophins, Preterm, Prematurity, Neonates

\section{Introduction}

With more than 100 billion neurons, human brain represents a massive network for information processing, storage and recall. The development and function of neurons is orchestrated by various growth factors, including neurotrophic factors. Among these are classical neurotrophins (NTs), the glial cell line-derived neurotrophic factor (GDNF) family of ligands (GDNF, neurturin (NRTN), artemin (ARTN), and persephin (PSPN)), and neuropoietic cytokines. The classical neurotrophin family comprises four structurally and functionally related molecules: nerve growth factor (NGF), brain-derived neurotrophic factor (BDNF), neurotrophin-3 (NT-3) and neurotrophin-4 (NT-4) [1]. NTs play a key role in proper brain development and are important mediators of synaptic plasticity. Indeed, NTs, once released by target cells, regulate the type and the number of afferent synapses by promoting the survival of discrete neuronal subpopulations. They also exert antiapoptotic activities in both the peripheral (sympathetic and sensory) and the central (cholinergic, dopaminergic, adrenergic) neurons as well as play important roles in higher neuronal functions and neurotransmitter expression [2].

The effects of NTs are mediated through signalling by two distinct classes of neurotrophin receptors (NTRs): the p75 pan-neurotrophin receptor (p75NTR) and the Trk family of tyrosine kinases [3]. The latter consists of three members: $\operatorname{TrkA}, \operatorname{TrkB}, \operatorname{TrkC}$, which preferentially bind to NGF, BDNF and NT-4, and NT-3, respectively $[4,5]$. Ligand binding to Trk receptors triggers activation of several intracellular signalling pathways, including ERK1/2, PI3K/Akt, Ras/ MAPK and phospholipase $\mathrm{C}$ pathways. Their

' Department of General Pathology, Pomeranian Medical University, Szczecin, Poland

*These authors equally contributed to the work.

${ }^{\dagger}$ Author for correspondence: Bogusław Machaliński, Department of General Pathology, Pomeranian Medical University in Szczecin, UI. Powstancow Wlkp. 72, 70-111 Szczecin, Poland, Tel: +48914661546; Fax: +48914661548; email: machalin@pum.edu.pl 
signalling cascades generally induce pro-survival and pro-growth cellular response [6]. p75NTR is the co-receptor that refines Trk affinity and specificity for NTs and augments Trk signalling. In the absence of Trks, p75NTR signals activate NF- $\kappa \mathrm{B}, \mathrm{JNK}$, and modulate RhoA activity, which are related to cell survival, death and neurite outgrowth, respectively [3].

Neurotrophins are widely expressed in nervous system during development and adulthood, but they also function in other systems, like immune and reproductive [7]. The expression of NTs is increased after injury, thus they have emerged as promising extrinsic factors to augment nerve regeneration [8]. Because of their unique characteristics, NTs have also been studied for their potential use in treatment; various trials with NTs showed enhanced neuronal survival, axonal regrowth, remyelination and synaptic plasticity [9]. In our previous studies we have shown that not only do NTs regenerate the damaged neurons, but they are also vital in protecting the existing ones [10]. Our promising results on cellular therapy in preterm infants indicate that certain growth factors, NTs included, could act as a preventive modality against prematurityrelated complications [11].

Preterm birth (<37 weeks of gestation) remains the leading cause of perinatal mortality and morbidity throughout the world [12]. Thirty percent of babies who survive though born before the 29th week of gestation show neurological, visual and intellectual impairment [13]. Premature infants are at high risk of developing specific conditions and diseases; these include brain injury (e.g. intraventricular haemorrhage; IVH, periventricular leukomalacia; PVL), retinopathy of prematurity (ROP), bronchopulmonary dysplasia (BPD), neonatal respiratory distress syndrome (RDS), necrotizing enterocolitis (NEC) and anaemia [14]. Neurotrophins concentrations were shown to be correlated with prematurity-related complications, birth weight, gestational age, or preterm birth in general $[15,16]$. Since NTs cross the immature blood brain barrier of premature infants [17], their application could provide neuroprotective and neuroregenerative effects, possibly ameliorating detrimental prematurityrelated complications, like IVH. The angiogenicspecific effect and neurotrophic activity of vascular endothelial growth factor (VEGF) also makes this trophic protein an attractive target for improving regenerative processes in a damaged central nervous system [18].
In our previous studies we showed that umbilical cord blood [UCB] transfusion to preterm infant results in beneficial increase in concentration of various growth factors, including NTs [11]. In this review we will focus on classical NTs clinical significance in preterm birth complications development. We will also focus on paracrine effects of stem/progenitor cells in regard to NTs and our novel cellular approach in preventing and ameliorating prematurity-related complications that our group has already successfully applied in previous clinical trials.

\section{Role of Neurotrophins in the Development of Nervous System}

Neuronal migration into the cortical mantle is largely complete by 25 weeks gestation, although, glial migration, that is mainly responsible for the expansion in cortical surface area, continues through the third trimester [19]. Brain cortical volume grows nearly fourfold between 29-35 weeks of gestation and it is a critical period for neuronal growth and migration [20]. Infants born at this gestation period have decreased cortical gray and white matter at term equivalent - secondary to apoptosis and neuronal atrophy - that impacts long term neurodevelopmental outcomes [21]. Brain injury (e.g. IVH and periventricular leukomalacia) constitutes the main dramatic complication in the perinatal period, with a potential of long-term neurodevelopmental disabilities [22]. In this matter, the earlier the child is born the higher the risk of brain injury - recent estimates suggest that nearly $40 \%$ of children born preterm will experience some level of neuronal development impairment [23]. From very-low-birth-weight [VLBW] infants (born $<32$ weeks and with weight $<1500$ g), approximately $10 \%$ have motor deficits and up to $60 \%$ have neurocognitive disabilities and behavioural disturbances [22,24]. It has been shown that premature birth also increases neuronal injury caused by inflammation and the hypoxic ischemic process [25]. Significant brain injury, or more subtle impairments in brain development, may underlie the development of major disabilities, such as cerebral palsy, mental retardation, autism spectrum disorder, deafness, or cortical visual impairment in the preterm neonate [26].

Development of the brain and nervous system is orchestrated by neurotrophins [27]; differences in NTs concentrations in term versus preterm infants seem to reflect compromised CNS 
development and early brain injury related to prematurity. Moreover, alterations in NTs expression can produce long lasting effects on neurotrophic processes (neurons/synapses numbers), which affect neuronal maturation and plasticity in later life [28].

Nerve growth factor, the first neurotrophin ever described, has long occupied a central position in developmental biology because of its action during a period of programmed cell death in embryonic and early postnatal life [29]. NGF is synthesized and secreted by sympathetic and sensory target organs, then it is captured in nerve terminals by receptor-mediated endocytosis and is transported through axons to neuronal cell bodies, there it acts to promote neuronal survival and differentiation [9,30]. NGF/TrkA knockout mice present with virtual absence of the superior cervical ganglia neurons by the first postnatal week, confirming the critical role of NGFITrkA signalling for the development of sympathetic neurons [31]. Although the role of NGF in developing brain is established, there are limited studies examining NGF levels in preterm infants. In the amniotic fluid NGF has been reported to raise with gestational age, additionally NGF levels in umbilical cord blood and plasma of preterm infants were significantly lower comparing to babies born at term [32]. According to Marx et al., low plasma NGF levels may be markers for the presence of either CNS abnormalities or infectious insults in utero or both [33]. Similarly, circulating BDNF reflects the degree of neuronal maturity in neonates [34], since, at this age, due to the immature blood-brain barrier, neurotrophin blood levels may also represent concentrations in the CNS. BDNF induces the growth and development of the central nervous system and is required for the survival of some primary sensory neurons during foetal development [35]. Umbilical cord blood BDNF concentrations increase with gestational age; low levels of BDNF in umbilical cord blood may indicate higher risk of neurodevelopmental complications, they may also be useful in predicting BDNF levels in the neonate and providing important clues for brain injury prevention $[34,36]$. NT-3 and NT-4 have been documented to act at early stages of neuronal development and to decrease after hypoxiaischaemia [37]. Studies have demonstrated the importance of NT-3 in the development of the CNS; for example, mice deficient in NT-3 lack proprioceptive and subsets of mechanoreceptive sensory neurons [38]. NT-3 plays an important role in the development of both the neuralcrest-derived peripheral nervous system and the central nervous system [39] and NT-3 levels are significantly decreased in preterm birth [40]. NT-3 mediates almost all neuron survival and differentiation-promoting activities; it diminishes neuronal loss, supports axonal elongation, and promotes angiogenesis and neurogenesis [9]. NT-4 binding to the TrkB receptor corresponds with the onset of neurogenesis in the neural tube during brain development [41]; NT-4 levels were found to be declined after preterm delivery [42].

Although the function of NTs in the developing nervous system contributes to the neurotrophic support of various neuronal populations, NTs mediate an extraordinary range of other functions, which are not amenable to simplified generalizations. During development, as has been shown experimentally, any manipulation that interrupts the flow of these neurotrophic factors, leads to the massive death of the affected neuronal pool [43]. Compromised neuronal development in preterm infants is reflected in altered neurotrophins expression, but NTs could also have a causative role in prematurity-related complications that expand beyond those strictly CNS-related ones.

\section{Preterm Birth Complications: Clinical Significance of Neurotrophins}

The cause of the prematurity complications is multifactorial and includes common pathogenic mechanisms such as ischemia/reperfusion and infection/inflammation, which promote the production of free radicals causative of oxidative stress. This leads to tissue damage and/or simplification, cell developmental arrest, scarring, fibrosis and in premature infant these detrimental effects are further enhanced by deprivation of protective maternal and placental factors and immaturity of the immune system [44]. Despite recent advances in neonatal intensive care medicine, intraventricular hemorrhage (IVH) remains a major cause of mortality and neurologic disability in premature infants, with few clinically effective treatments [45]. The pathogenesis of IVH is ascribed to the intrinsic fragility of germinal matrix vasculature - it exhibits accelerated angiogenesis, which orchestrates formation of nascent vessels that lack pericytes, display immature basal lamina low in fibronectin, and have astrocyte endfeet coverage deficient in GFAP [46]. Since angiogenesis holds a prominent role in the pathogenesis of IVH (and 
other prematurity-related complications), not only neurotrophins are regarded as crucial, but so is VEGF. Data demonstrate that the developing brain responds to chronic sublethal hypoxia with increases in permeability and angiogenesis and suggest that VEGF mediates this response [47]. In animal models, antagonism of VEGF decreased the occurrence of brain haemorrhage [48], but the exact role of VEGF in IVH in preterm neonates is yet to be clarified.

Neurotrophins act together with VEGF to enhance brain repair after hypoxia and/or ischemia. Here, particularly significant role is attributed to BDNF. In $2017 \mathrm{Ahn}$ et al. suggested that BDNF secreted by transplanted mesenchymal stem cells might be a critical paracrine factor mediating their neuroprotective effects after severe IVH in the newborn rats [49]. In particular, the group attributed protective role against severe IVH-induced injury to BDNF downstream cell signalling involved in cell survival and significantly reduced neuronal death both in vitro after excitotoxic insults and in vivo after focal ischemia and bacterial meningitis. Thus, anti-inflammatory and antiapoptotic effects might be other potential neuroprotective mechanisms of BDNF. The lower levels of umbilical cord BDNF and NGF in preterm pregnancies may provide clues to predict cognitive deficits in children: preterm infants who develop moderate or severe peri/ intra-ventricular haemorrhage $[\mathrm{P} / \mathrm{IVH}]$ have lower umbilical BDNF levels than those with mild P/IVH or no cerebral injury [27].

Children born preterm are at higher risk for visual impairment due to cerebral visual impairment, which is caused by damage to the geniculocalcarine pathways, and is related to the severity of white matter injury [50,51]. Nowadays, retinopathy of premature [ROP] is a leading cause of childhood blindness worldwide [52]. ROP proceeds following an initial phase of degeneration of the retinal microvasculature (vaso-obliteration) that is associated with cessation of progression of vascular growth toward the retinal periphery resulting in ischemic retina. In the subsequent phase of the disease, the ensuing retinal ischemia predisposes to abnormal compensatory neovascularization [53]. Various risk factors have been linked to the development of ROP include low birth weight, low gestational age, supplemental and oxygen therapy [54]. Although NTs are heavily involved in growth, survival, proliferation, and migration of neurons in the developing brain and retina, there are limited data whether premature birth and development of ROP is associated with alteration in neurotrophins. It is recognized that neural factors provide guidance cues for both neurons and vascular cells, suggesting the importance of neurovascular interactions in retinal and vascular development [55]. The role of BDNF in ROP is supported by a recent study that examined single nucleotide polymorphism in a large cohort of premature infants with levels of ROP severity: two intronic variants in BDNF gene (rs7934165 and rs2049046) were associated with severe ROP [56]. BDNF is expressed in the visual cortex and retina during development and plays an important role in development of visual plasticity. It was noted that BDNF concentrations were decreased in infants who developed ROP and trended lower in those with more severe ROP [15]. Association between ROP and lower levels of NT-4 was observed as early as postnatal day 3 (D3), it continued to be low on D7 [57]. It is hypothesized that lower serum concentrations of BDNF and NT-4 in infants with ROP compared to infants without ROP may perfectly reflect glial and neuronal loss associated with ROP [57]. BDNF and NT-4 play a role in the regulation of intraocular inflammation [57] and they have been demonstrated to promote retinal ganglion cell survival after injury [58].

Angiogenesis is one of the crucial pathophysiological processes for both IVH and ROP. In fact, it seems that the vascular system links most, if not all prematurity complications $[59,60]$. The fragility of underdeveloped vascular system is a point from which the organism tries to escape by means of, rather chaotic, angiogenesis [61]. During the embryonic development, vessel formation and maturation is a very dynamic process, involving numerous growth factors, including neurotrophins that cooperate with one of angiogenesis' 'master regulators', VEGF $[62,63]$. BDNF and NGF play an important role in the regulation of angiogenesis in tissues $[64,65]$, in fact circulating BDNF may be secreted by vascular endothelial cells [66] and is involved in VEGF expression regulation [67]. NGF acts as an angiogenic factor by contributing to the maintenance, survival and function of endothelial cells by autocrine and/ or paracrine mechanisms. NGF, BDNF and NT-3 might have the potential to greatly increase angiogenesis in pathological situations [68]. One of the possible mechanisms for angiogenesis through neurotrophins could be that BDNF/ NGF-TrkB/TrkA signaling cascade increases 
hypoxia-inducible factor 1-alpha (HIF-1 $\alpha)$ and may regulate VEGF through mitogen-activated protein kinases/extracellular signal-regulated kinases (MAPK/ERK) and protein kinase $\mathrm{B}$ (PKB) pathways in the placenta in very early pregnancy [69].

Pathogenesis of prematurity-related disorders is not limited to angiogenesis only, but other pathological processes also provide a link between preterm birth complications. In fact, BDNF serves as a connection between neonatal lung injury and motoneuron survival. In cultured rat embryonic motoneurons, BDNF has been shown to support motoneuron survival by preventing neuronal nitric oxide synthase (NOS) expression [70]. Moreover, not only does BDNF appear to down-regulate NOS expression, but exogenous $\mathrm{NO}$ administration clearly inhibits BDNF secretion in embryonic rat hippocampal neurons. BDNF/NO interactions may also contribute to airway homeostasis in the developing respiratory system and modulate lung injury responses [71]. BDNF can enhance $\mathrm{Ca}^{2+}$, cell proliferation, migration and airway remodelling in general. These processes are equally important in the developing airway, perhaps more so given that a stiffer, thicker airway can lead to severe respiratory decompensation in children. Use of antenatal steroids (ANS) for pulmonary maturation is associated with improved neurodevelopmental outcomes in preterm infants. ANS mediated neuroprotection may be through increased BDNF synthesis or alternately, neuronal maturation following ANS may result in increased BDNF concentrations [42]. Increasing evidence from studies of airway hyper reactivity in asthmatic patients suggests that also NGF may serve as a link between airway inflammation and airway hyper responsiveness. In infants with respiratory syncytial virus infection who are highly predisposed to airway hyper reactivity elevated levels of NGF were found [71].

It has been suggested that in preterm infants growth factors balance is skewed toward inflammation; there is evidence that many of the long-term problems that account for much of the morbidity of prematurity are inflammatory in nature [72]. One of them is necrotizing enterocolitis (NEC), in which NTs seem to also play a vital part. Neurotrophins are likely to be among the factors that influence the differentiation and phenotypic expression of precursor crest-derived cells in the gut [73]. There is evidence for the presence of functional neurotrophin receptors in the developing enteric nervous system [74]. NT-3, the most abundant neurotrophin in the nervous system during early phases of development, is the most relevant for developing enteric nervous system, where it acts simultaneously with GDNF [75]. Experimental studies have shown the protective effect of GDNF and ciliary neurotrophic factor (CNTF) in enteric neuronal survival, which seem to cooperate with classical neurotrophins in pathological and physiological states of gut development [76]. NGF has been shown to act as an inflammatory mediator associated with functional activities of cells that include immune and endocrine systems [29]. Umbilical cord blood NT-3 levels were significantly decreased in the presence of placental inflammation [77]. In both animal and human models, BDNF concentrations are decreased in subjects exposed to prenatal stress and infections [78].

Clinical significance of neurotrophins in prematurity-related complications is not limited to CNS only, but they play a role in angiogenesis, inflammation and probably a vast spectrum of other, still not fully elucidated, pathological processes which underlie the development of detrimental disorders that preterm infants are facing.

\section{Stem/Progenitor Cells in Premature Infants}

Because the available treatment options for prematurity-related complications are still quite limited, the vista of using stem/progenitor cells (SPCs) for tissue repair is seen as big hope for immature preterm infants among perinatologists. Preterm infants are quite unique when it comes to SPCs, as the number of circulating SPCs has been correlated with prematurity-related complications and UCB from preterm babies seems to be a particularly good source of SPCs. In the next chapter we will discuss the circulating SPCs and their paracrine effects and the novel cellular approaches which our team has successfully applied in various clinical trials.

\section{Circulating Stem/Progenitor Cells}

Stem/progenitor cells (SPCs) are able to give rise to multiple cell types and are widely used in regenerative medicine. The cell membrane protein CD34 is an important marker of early SPCs, and the CD34+ cell population is thought to comprise early hematopoietic SPCs and endothelial progenitor cells [79]. 
Evidence suggests that a portion of SPCs circulates in the peripheral blood to maintain a balance between SPCs populations in various anatomic areas of the living organism [80]. The number of these cells increases during organ and tissue injury, e.g. heart infarct, stroke, heavy burns, or liver or skeletal muscle damage. During prenatal development, various types of stem cells migrate, proliferate and differentiate to form tissues and organs. Tissue and peripheral blood SPCs pools are in dynamic equilibrium with each other, allowing stem cells to migrate between extravascular sites or marginal pools and the circulation. We have recently shown that the number of primitive SPCs circulating in UCB is a valuable predictor of prematurity complications in preterm newborns [81].

Also a population of bone marrow (BM) derived circulating early and late endothelial progenitor cells (EPCs) is inversely associated with the Apgar score of preterm infants [82]. As we have shown, high number of circulating early-EPCs is associated with higher risk of RDS, ROP, BPD, and infections in newborns, however, the number of circulating EPCs is not an independent predictor of prematurity complications [83]. The number of circulating EPCs in full-term infants was maintained at a constant, relatively low-level, what may result from the maturation of organs undertaking adequately their physiological functions. On the contrary, EPC concentration gradually decreases in preterm infants over time [84,85]. The elevation of VEGF expression in preterm infants may contribute to an increase in the number of circulating EPCs and physiological development of vascularised organs [86]. SPCs migration is mediated by VEGF, SDF-1, and HGF, the most important chemotactic factors critical for stem cell motility, homing, and mobilisation; their concentrations in UCB are significantly lower in preterm than in fullterm infants [87]. Data are accumulating that EPC enumeration represents a viable strategy for assessing the regenerative capacity of the vascular system [88]. Therefore, a higher concentration of these cells in the UCB of preterm infants, reflects the prematurity of the infants' vascularised organs, but also opens up a possibility of UCB use for cellular therapy, which will be discussed later.

\section{Paracrine Effects of Circulating Stem/ Progenitor Cells}

One of the easily accessible and quite attractive sources of unique SPCs with immature characteristics is umbilical cord blood [89]. The beneficial effects of stem cell-based therapy may be based on the trophic activity of SPCs, which produce various cytokines, including NTs, regulating growth, differentiation, and migration of neural SPCs. Numerous studies suggests that UCB cell-induced neuroprotection involves anti-inflammatory and immunomodulatory effects, and that neurotrophic factors act through paracrine and autocrine interactions between transplanted cells and the neural microenvironment [90-92]. We have recently shown that CD34+ SPCs express NTs (BDNF, NT-3, NT-4, NGF) and VEGF at higher levels than unsorted nuclear cells (NCs) [79]. Apart from classical NTs, three SPC populations (lineage-negative, CD34+, CD133+) also express the neuropoietic cytokines CDNF, MANF, and PEDF. These cytokines demonstrate neuroprotective qualities, similarly to VEGF, which has angiogenic and neurotrophic activities. UCB-derived SPCs also possess the neurotrophin receptors p75NTR, TrkA, TrkB, TrkC, GRFa1, and GFRa2 at the transcriptional and posttranscriptional levels [94]. Neurotrophin receptors could be involved in the recruitment of SPCs to specific sites, like is a case with BDNF and TrkB. Production of various growth factors (including NTs) by UCB-derived SPCs can be increased by exposure of these cells to stress conditions [71], a phenomenon which our group decided to apply in cellular therapy for neurodegenerative disorders. As lineage negative [Lin-] cells exposed to stress conditions (short-term serum-free culture) show enhanced NTs production, this suggests using "stressed" Lin- cells for their enhanced neuroregenerative and neuroprotective role. Therefore, we are now conducting a trial in which Lin- transplantation to non-optimal microenvironment [cerebrospinal fluid] might boost NTs production and be clinically beneficial; initial results of our study are encouraging (manuscript in preparation). It has been also suggested that short-term priming by BDNF may enhance the trophic activity of SPCs [95,96]. The influence consists of diminishing apoptosis in these cells, modulating antioxidants and increasing NT-3 expression. These findings support the hypothesis that pre-treatment of SPCs could be beneficial and may be used as an auxiliary strategy for improving the properties of SPCs.

\section{Novel Approach of Adjuvant Cell Therapy in Immature Preterm Babies}

Independently of the cause of preterm labour, the infant within seconds sustains a substantial loss 
of blood and its components, as $50 \%$ or more of his blood volume with circulating SPCs pool is removed with umbilical cord and placental blood vessels [81]. Due to more advanced, physiological development, the cord blood and placenta of full term infant contain approximately $23-27 \%$ more total blood volume with relatively lower SPCs numbers [97]. We have shown that preterm UCB has significantly greater number of SPCs with increased clonogenicity and earlier cellular differentiation compared to full term UCB [98]; this has provided new treatment possibilities that could be of great importance during the neonatal period. Although cell-based therapy is relatively novel approach to the treatment of prematurity complications, our encouraging initial experience together with new data derived from novel animal studies widen the horizons for this innovative strategy [11,99-101].

We have proposed to replace SPCs lost during delivery by applying autologous whole UCB transfusion in the first days after birth. This technique might provide a cocktail of soluble proteins (including neurotrophins) and restore the initial number of SPCs that provide paracrine effects, resulting in the reestablishment of physiologic conditions for newborn organ development [101]. Clinical outcomes of our study are encouraging - autologous UCB infusion was no less effective and no less safe than allogeneic RBC transfusion. We have also designed a collection bag system, which makes UCB collection easier [102]. Our most recent study showed that the array of neurotrophins and growth factors produced by UCB cells is a major advantage of UCB over RBC transfusion [11]. We believe that further studies on larger cohorts of infants are necessary to fully elucidate all benefits and possible drawbacks of UCB transfusion. Because most prematurity complications result from underdeveloped vascular and nervous system, if autologous UCB transfusion results in increased concentrations of growth factors involved in angiogenesis, angiopoiesis, and neovascularisation, early administration of UCB to preterm infants could be valuable preventive treatment. It might compensate for the anaemia, but also provide vital growth factors, which prevent otherwise chaotic angiogenesis and play a role in neuroprotection and neuroregeneration.

\section{Conclusion}

Neurotrophins promote survival and reduce apoptosis in many populations of neurons, they also play essential roles in axon growth during development, higher neuronal functions, morphologic differentiation and neurotransmitter expression. NTs are also involved in preterm birth complications and could be used therapeutically, as they augment neuroprotection and neuroregeneration. Neurotrophins action is not limited to neuronal tissue, as their role in angiogenesis and inflammation has also been proposed. Abrupt removal of maternal passage and placenta sources could cause the sharp decline in NTs levels after birth. Novel cellular approaches involving the use of stem/progenitor cells producing various growth factors, including neurotrophins, are emerging to prevent prematurity-related complications. Further research is needed to elucidate neurotrophins' role in physiology and pathology, in order to warrant better therapeutic and preventive options for prematurity-related complications.

\section{Funding}

This work was supported by The National Centre for Research and Development (grant number STRATEGMED1/234261/2NCBR/2014).

\section{References}

1. Barde YA. The nerve growth factor family. Prog. Growth. Factor. Res 2(4), 237-248 (1990).

2. Chao MV. Trophic factors, An evolutionary cul-de-sac or door into higher neuronal function? J. Neurosci. Res 59(3), 353 (2000).

3. Roux PP, Barker PA. Neurotrophin signaling through the p75 neurotrophin receptor. Prog. Neurobiol 67(3), 203-233 (2002).

4. Reichardt LF. Neurotrophin-regulated signalling pathways. Philos. Trans. R. Soc. Lond. B. Biol. Sci 361(1473), 1545-1564 (2006).
5. Wu YS, Chiou YJ, Huang TL. Associations between Serum Brain-Derived Neurotrophic Factors and Bipolar Disorder. Neuropsychiatry 7(6), 968-973 (2017).

6. Hillis J, ODwyer M, Gorman AM. Neurotrophins and B-cell malignancies. Cell. Mol. Life. Sci 73(1), 41-56 (2016).

7. Ivanisevic L, Saragovi HU. Neurotrophins A2 - Kastin, Abba J. Handbook of Biologically Active Peptides (Second Edition). Boston, Academic Press; 1639-1646 (2013).

8. Machaliñski $B$, Łażewski-Banaszak $P$, Dąbkowska E, et al. Rola czynników neurotroficznych w procesach regeneracji układu nerwowego. Neurologia. $i$. Neurochirurgia. Polska 46(6), 579-590 (2012).

9. Lykissas MG, Batistatou AK, Charalabopoulos $\mathrm{KA}$, et al. The role of neurotrophins in axonal growth, guidance, and regeneration. Curr. Neurovasc. Res 4(2), 143-151 (2007).

10. Machalinska A, Kawa M, Pius-Sadowska E, et al. Long-term neuroprotective effects of NT-4-engineered mesenchymal stem cells injected intravitreally in a mouse model of acute retinal injury. Invest. Ophthalmol. Vis. Sci 54(13), 8292-8305 (2013). 
11. Kotowski M, Litwinska Z, Klos $P$, et al. Autologous cord blood transfusion in preterm infants - could its humoral effect be the key to control prematurity-related complications? A preliminary study. J. Physiol. Pharmacol 68(6), 921-927 (2017).

12. Kinney MV, Lawn JE, Howson CP, et al. 15 million preterm births annually, what has changed this year? Reprod. Health 9(1) (2012).

13. Pasquier JC, Picaud JC, Rabilloud M, et al. Neonatal outcomes after elective delivery management of preterm premature rupture of the membranes before 34 weeks' gestation (DOMINOS study). Eur. J. Obstet. Gynecol. Reprod. Biol 143(1), 18-23 (2009).

14. Hall ES, Greenberg JM. Estimating community-level costs of preterm birth. Public. Health 141, 222-228 (2016).

15. Rao R, Mashburn CB, Mao J, et al. BrainDerived Neurotrophic Factor in Infants $<32$ Weeks Gestational Age, Correlation With Antenatal Factors and Postnatal Outcomes. Pediatr. Res 65(5), 548-552 (2009).

16. Bachnas MA, Mose JC, Effendi JS, et al. Influence of antenatal magnesium sulfate application on cord blood levels of brainderived neurotrophic factor in premature infants. J. Perinat. Med 42(1), 129-134 (2014).

17. Laterra J, Goldstein GW. Development of the Blood-Brain Barrier. Fetal. Neonatal. Physiol 1699-706 (2004).

18. Bogaert $E$, Van Damme $P$, Poesen $K$, et al. VEGF protects motor neurons against excitotoxicity by upregulation of GluR2. Neurobiol. Aging 31(12), 2185-2191 (2010).

19. Barkovich AJ, Raybaud CA. Neuroimaging in disorders of cortical development. Neuroimag. Clini 14(2), 231-254 (2004).

20. Nagy Z, Lagercrantz H, Hutton C. Effects of Preterm Birth on Cortical Thickness Measured in Adolescence. Cereb. Cortex 21(2), 300-306 (2010)

21. Counsell SJ. Magnetic resonance imaging of preterm brain injury. Fetal. Neonatal 88(4), 269-274 (2003).

22. Woodward LJ, Edgin JO, Thompson D, et al. Object working memory deficits predicted by early brain injury and development in the preterm infant. Brain 128(11), 25782587 (2005).

23. Mwaniki MK, Atieno M, Lawn JE, et al. Long-term neurodevelopmental outcomes after intrauterine and neonatal insults, a systematic review. Lancet 379(9814), 445452 (2012).

24. Holsti L, Grunau RVE, Whitfield MF. Developmental Coordination Disorder in Extremely Low Birth Weight Children at Nine Years. J. Dev. Behav. Pediatr 23(1), 9-15 (2002).
25. Baburamani AA, Ek CJ, Walker DW, et al. Vulnerability of the developing brain to hypoxic-ischemic damage, contribution of the cerebral vasculature to injury and repair? Front. Physiol 3, 424 (2012).

26. Saigal S, Doyle LW. An overview of mortality and sequelae of preterm birth from infancy to adulthood. Lancet 371(9608), 261-269 (2008).

27. Dhobale M. Neurotrophins, Role in adverse pregnancy outcome. Int. J. Dev. Neurosci 37 8-14 (2014).

28. Vicario-Abejón C, Owens D, McKay R, et al. Role of neurotrophins in central synapse formation and stabilization. Nat. Rev. Neurosci 3(12), 965-974 (2012).

29. Berry A, Bindocci E, Alleva E. NGF, Brain and Behavioral Plasticity. Neural. Plast 2012, 1-9 (2012).

30. Zhu Z, Wu C, Liu Z, et al. Association between Serum Nerve Growth Factor Levels and Depression in Stroke Patients. Neuropsychiatry 7(6), 1061-1068 (2017).

31. Smeyne RJ, Klein R, Schnapp A, et al. Severe sensory and sympathetic neuropathies in mice carrying a disrupted Trk/NGF receptor gene. Nature 368(6468), 246-249 (1994).

32. Toti $P$, Ciarmela $P$, Florio $P$, et al. Human placenta and fetal membranes express nerve growth factor mRNA and protein. $J$. Endocrinol. Invest 29(4), 337-341 (2006).

33. Marx CE, Vance BJ, Jarskog LF, et al. Nerve growth factor, brain-derived neurotrophic factor, and neurotrophin-3 levels in human amniotic fluid. Am. J. Obstet. Gynecol 181(5), 1225-1230 (1999).

34. Chouthai NS, Sampers J, Desai N, et al. Changes in Neurotrophin Levels in Umbilical Cord Blood From Infants With Different Gestational Ages and Clinical Conditions. Pediatr. Res 53(6), 965-969 (2003).

35. Erickson JT, Brosenitsch TA, Katz DM. BrainDerived Neurotrophic Factor and Glial Cell Line-Derived Neurotrophic Factor Are Required Simultaneously for Survival of Dopaminergic Primary Sensory NeuronsIn Vivo. J. Neurosci 21(2), 581-589 (2001).

36. Dhobale M, Mehendale S, Pisal H, et al Association of brain-derived neurotrophic factor and tyrosine kinase $B$ receptor in pregnancy. Neurosci 216, 31-37 (2012).

37. Nikolaou KE, Malamitsi-Puchner A, Boutsikou T, et al. The Varying Patterns of Neurotrophin Changes in the Perinatal Period. Annal. N. Y. Acad. Sci 1092(1), 426433 (2006).

38. Klein R, Silos-Santiago I, Smeyne RJ, et al. Disruption of the neurotrophin-3 receptor gene trkC eliminates la muscle afferents and results in abnormal movements. Nature 368(6468), 249-251 (1994).
39. Chalazonitis A. Neurotrophin-3 in the development of the enteric nervous system. Prog. Brain. Res 243-263 (2004)

40. Matoba N, Yu Y, Mestan K, et al. Differential Patterns of 27 Cord Blood Immune Biomarkers Across Gestational Age. Pediatrics 123(5), 1320-1328 (2009).

41. Hennigan A, O'Callaghan RM, Kelly ÁM. Neurotrophins and their receptors, roles in plasticity, neurodegeneration and neuroprotection. Biochem. Soc. Trans 35(2), 424-427 (2007).

42. Kumar $P$, Venners SA, Fu L, et al. Association of antenatal steroid use with cord blood immune biomarkers in preterm births. Early. Hum. Dev 87(8), 559-564 (2011).

43. Holtzman DM, Mobley WC. Neurotrophic factors and neurologic disease. West. J. Med 161(3), 246-254 (1994).

44. Elovitz MA, Mrinalini C, Sammel MD. Elucidating the Early Signal Transduction Pathways Leading to Fetal Brain Injury in Preterm Birth. Pediat. Res 59(1), 50-55 (2006).

45. Ballabh P. Pathogenesis and prevention of intraventricular hemorrhage. Clin. Perinatol 41(1), 47-67 (2014)

46. Ballabh P. Intraventricular Hemorrhage in Premature Infants, Mechanism of Disease. Pediatr. Res 67(1), 1-8 (2010).

47. Jin $K$, Zhu Y, Sun Y, et al. Vascular endothelial growth factor (VEGF) stimulates neurogenesis in vitro and in vivo. Proceedings of the National Academy of Sciences of the United States of America 99(18), 11946-11950 (2002).

48. Jiang $S$, Xia R, Jiang $Y$, et al. Vascular endothelial growth factors enhance the permeability of the mouse blood-brain barrier. PLoS. One 9(2), e86407 (2010).

49. Ahn SY, Chang YS, Sung DK, et al. Pivotal Role of Brain-Derived Neurotrophic Factor Secreted by Mesenchymal Stem Cells in Severe Intraventricular Hemorrhage in Newborn Rats. Cell. Transplant 26(1), 145156 (2017)

50. Cioni G, Fazzi B, Coluccini M, et al. Cerebral visual impairment in preterm infants with periventricular leukomalacia. Pediatr. Neurol 17(4), 331-338 (1997).

51. Lanzi G, Fazzi E, Uggetti C, et al. Cerebral Visual Impairment in Periventricular Leukomalacia*. Neuropediatr 29(3), 145-150 (1998).

52. Zin A, Gole GA. Retinopathy of PrematurityIncidence Today. Clin. Perinatol 40(2), 185200 (2013).

53. Berkowitz BA, Roberts R, Penn JS, et al. High-Resolution Manganese-Enhanced MRI of Experimental Retinopathy of Prematurity. Invest. Ophthalmol. Vis. Sci 
48(10), 4733 (2007)

54. Sapieha P, Joyal J-S, Rivera JC, et al. Retinopathy of prematurity, understanding ischemic retinal vasculopathies at an extreme of life. J. Clin. Invest 120(9), 3022-3032 (2010).

55. Fukushima Y, Okada M, Kataoka $\mathrm{H}$, et al. Sema3E-PlexinD1 signaling selectively suppresses disoriented angiogenesis in ischemic retinopathy in mice. J. Clin. Invest 121(5), 1974-1985 (2011).

56. Hartnett ME, Morrison MA, Smith S, et al. Genetic Variants Associated With Severe Retinopathy of Prematurity in Extremely Low Birth Weight Infants. Invest. Ophthalmol. Vis. Sci 55(10), 6194 (2014).

57. Sood BG, Madan A, Saha S, et al. Perinatal Systemic Inflammatory Response Syndrome and Retinopathy of Prematurity. Pediatr. Res 67(4), 394-400 (2010).

58. Perez M-TR, Caminos E. Expression of brain-derived neurotrophic factor and of its functional receptor in neonatal and adult rat retina. Neurosci. Let 183(1-2), 96-99 (1995).

59. Lassus $P$, Turanlahti $M$, Heikkil ÄP, et al. Pulmonary Vascular Endothelial Growth Factor and Flt-1 in Fetuses, in Acute and Chronic Lung Disease, and in Persistent Pulmonary Hypertension of the Newborn. Am. J. Resp. Crit. Care. Med 164(10), 1981-1987 (2001).

60. Shimi MSE, Hassanein SMA, Mohamed MH, et al. Predictive value of vascular endothelial growth factor in preterm neonates with intraventricular haemorrhage. J. Matern. Fetal. Neonatal. Med 25(9), 1586-1590 (2012).

61. Potente $M$, Gerhardt $H$, Carmeliet P. Basic and Therapeutic Aspects of Angiogenesis. Cell 146(6), 873-887 (2011).

62. Koch S, Claesson-Welsh L. Signal Transduction by Vascular Endothelial Growth Factor Receptors. Cold. Spring. Harb. Perspect. Med 2(7), a006502 (2012).

63. Kermani P, Hempstead B. Brain-Derived Neurotrophic Factor, A Newly Described Mediator of Angiogenesis. Trends. Cardiovasc. Med 17(4), 140-143 (2007).

64. Jadhao CS, Bhatwadekar AD, Jiang $Y$, et al. Nerve Growth Factor Promotes Endothelial Progenitor Cell-Mediated Angiogenic Responses. Invest. Ophthalmol. Vis. Sci 53(4), 2030 (2012).

65. Jiang $H$, Liu Y, Zhang $Y$, et al. Association of plasma brain-derived neurotrophic factor and cardiovascular risk factors and prognosis in angina pectoris. Biochem. Biophys. Res. Commun 415(1), 99-103 (2011).

66. Nakahashi T, Fujimura H, Altar CA et al. Vascular endothelial cells synthesize and secrete brain-derived neurotrophic factor. FEBS. Let 470(2), 113-137 (2000).

67. Nakamura K, Martin KC, Jackson JK, et al.
Brain-Derived Neurotrophic Factor Activation of TrkB Induces Vascular Endothelial Growth Factor Expression via Hypoxia-Inducible Factor-1 a in Neuroblastoma Cells. Cancer. Res 66(8), 4249-4255 (2006).

68. Blais $M$, Lévesque $P$, Bellenfant $S$, et al. Nerve Growth Factor, Brain-Derived Neurotrophic Factor, Neurotrophin-3 and Glial-Derived Neurotrophic Factor Enhance Angiogenesis in a Tissue-EngineeredIn VitroModel. Tissue. Eng. Part. A 19(15-16), 1655-1664 (2013).

69. Shi G-X, Andres DA, Cai W. Ras Family Small GTPase-mediated Neuroprotective Signaling in Stroke. Cent. Nerv. Syst. Agents. Med. Chem 11(2), 114-137 (2011)

70. Estévez AG, Spear N, Manuel SM, et al. Nitric Oxide and Superoxide Contribute to Motor Neuron Apoptosis Induced by Trophic Factor Deprivation. J. Neurosci 18(3), 923-931(1988) .

71. Yao Q, Zaidi SI, Haxhiu MA, et al. Neonatal Lung and Airway Injury, A Role for Neurotrophins. Semin. Perinatol 30(3), 156162 (2006).

72. Cai S-y, Liu Z-h, Peng G-l, et al. The Effects of Exercise Therapy on Motor Behaviors of Preterm Infant Rats Induced by Intrauterine Inflammation. Neuropsychiatry 7(5), 667-674 (2017).

73. Gershon MD, Chalazonitis Ae, Rothman TP. From neural crest to bowel, Development of the enteric nervous system. J. Neurobiol 24(2), 199-214 (1993).

74. Sternini C, Su D, Arakawa J, et al. Cellular localization of pan-trk immunoreactivity and trkC mRNA in the enteric nervous system. J. Comp. Neurol 368(4), 597-607 (1996).

75. Chalazonitis A, Rothman TP, Chen J, et al. Neurotrophin-3 induces neural crest-derived cells from fetal rat gut to develop in vitro as neurons or glia. The Journal of Neuroscience. 1994;14(11), 6571-84.

76. Fichter M, Klotz M, Hirschberg DL, et al. Breast milk contains relevant neurotrophic factors and cytokines for enteric nervous system development. Mol. Nutr. Food. Res 55(10), 1592-1596 (2011).

77. Shang A-J, Hong S-Q, Xu Q, et al. NT3-secreting human umbilical cord mesenchymal stromal cell transplantation for the treatment of acute spinal cord injury in rats. Brain. Res 1391, 102-113 (2011).

78. Gilmore JH, Jarskog LF, Vadlamudi S. Materna infection regulates BDNF and NGF expression in fetal and neonatal brain and maternal-fetal unit of the rat. J. Neuroimmunol 138(1-2), 4955 (2003).

79. Paczkowska E, Piecyk K, Luczkowska K, et al. Expression of neurotrophins and their receptors in human $\mathrm{CD} 34+$ bone marrow cells. J. Physiol. Pharmacol 67(1), 151-159 (2016).

80. Machalińska A, Baumert B, Kuprjanowicz L, et al. Potential Application of Adult Stem Cells in Retinal Repair-Challenge for Regenerative Medicine. Curr. Eye. Res 34(9), 748-760 (2009).

81. Kotowski M, Safranow K, Kawa MP, et al. Circulating hematopoietic stem cell count is a valuable predictor of prematurity complications in preterm newborns. BMC. Pediatr 12(1) (2012).

82. Chong MS, Ng WK, Chan JK. Concise Review, Endothelial Progenitor Cells in Regenerative Medicine, Applications and Challenges. Stem. Cell. Transl. Med 5(4), 530-538 (2016).

83. Safranow K, Kotowski M, Lewandowska J, et al. Circulating endothelial progenitor cells in premature infants, is there an association with premature birth complications? J. Perinat. Med 40(4), 455-462 (2012).

84. Machalinska A, Safranow K, Dziedziejko V et al. Different Populations of Circulating Endothelial Cells in Patients with Age-Related Macular Degeneration, A Novel Insight into Pathogenesis. Invest. Opthalmol. Vis. Sci 52(1), 93 (2011).

85. Real C, Caiado F, Dias S. Endothelial Progenitors in Vascular Repair and Angiogenesis, How Many are Needed and What to do? Cardiovasc. Hematol. Disord. Drug. Targets 8(3), 185-192 (2008).

86. Young PP, Hofling AA, Sands MS. VEGF increases engraftment of bone marrowderived endothelial progenitor cells (EPCs) into vasculature of newborn murine recipients. Proc. Natl. Acad. Sci. U. S. A 99(18), 11951-11956 (2002).

87. Lapidot T. How do stem cells find their way home? Blood 106(6), 1901-1910 (2005).

88. Fischer-Rasokat U, Dimmeler S. Endothelial Progenitor Cells for Cardiac Regeneration. Stem. Cell. Transplant 177-195 (2006).

89. Kögler G, Sensken S, Airey JA, et al. A New Human Somatic Stem Cell from Placental Cord Blood with Intrinsic Pluripotent Differentiation Potential. J. Exp. Med 200(2), 123-135 (2004).

90. Arien-Zakay H, Lecht S, Bercu MM, et al. Neuroprotection by cord blood neural progenitors involves antioxidants, neurotrophic and angiogenic factors. Exp. Neurol 216(1), 83-94 (2009).

91. Jiang L, Womble T, Saporta S, et al. Human Umbilical Cord Blood Cells Decrease Microglial Survival In Vitro. Stem. Cell. Dev 19(2), 221-228 (2010).

92. Leonardo CC, Hall AA, Collier LA, et al. Human umbilical cord blood cell therapy blocks the morphological change and recruitment of CD11b-expressing, isolectin-binding proinflammatory cells after middle cerebral artery occlusion. J. Neurosci. Res 88(6),12131222 (2009).

93. Paczkowska E, Piecyk K, Luczkowska K, et al. Expression of neurotrophins and their 


\section{Review Bogusław Machaliński}

receptors in human $\mathrm{CD} 34+$ bone marrow cells. J. Physiol. Pharmacol 67(1), 151-159 (2016).

94. Paczkowska E, Kaczynska K, Pius-Sadowska $\mathrm{E}$, et al. Humoral activity of cord bloodderived stem/progenitor cells, implications for stem cell-based adjuvant therapy of neurodegenerative disorders. PLoS. One 8(12), e83833 (2013).

95. Sanberg PR, Willing AE, Garbuzova-Davis $\mathrm{S}$, et al. Umbilical Cord Blood-Derived Stem Cells and Brain Repair. Ann. N. Y. Acad. Sci 1049(1), 67-83 (2005).

96. McGuckin CP, Forraz N, Allouard Q, et al. Umbilical cord blood stem cells can expand hematopoietic and neuroglial progenitors in vitro. Exp. Cell. Res 295(2), 350-359 (2004).

97. Machaliński B. Stem Cell Therapies in Neonatology. Stem. Cell. Biol. Regen. Med 1(1), 179-190 (2014).

98. Machalinska A, Modrzejewska M, Kotowski $M$, et al. Circulating stem cell populations in preterm infants, implications for the development of retinopathy of prematurity. Arch. Ophthalmol 128(10), 1311-1319 (2010).

99. Meier C, Middelanis J, Wasielewski B, et al. Spastic Paresis After Perinatal Brain Damage in Rats Is Reduced by Human Cord Blood Mononuclear Cells. Pediatr. Res 59(2), 244-249 (2006)
100. Pierro $\mathrm{M}$, lonescu L, Montemurro $\mathrm{T}$, et al. Short-term, long-term and paracrine effect of human umbilical cord-derived stem cells in lung injury prevention and repair in experimental bronchopulmonary dysplasia. Thorax 68(5), 475-484 (2012).

101.Rudnicki J, Kawa MP, Kotowski M, et al. Clinical Evaluation of the Safety and Feasibility of Whole Autologous Cord Blood Transplant as a Source of Stem and Progenitor Cells for Extremely Premature Neonates, Preliminary Report. Exp. Clin. Transplant 13(6), 563-572 (2015).

102. Machaliński B, Bartkowiak A, Rudnicki J, et al. Inventors Set for umbilical cord blood collection. Poland (2016). 